\title{
Exact BER Calculation of Asynchronous DS-CDMA Systems Communicating over Hoyt Channels
}

\author{
Xiang Liu and Lajos Hanzo \\ School of Electronics and Computer Science, University of Southampton, SO17 1BJ, UK \\ lh@ecs.soton.ac.uk,www-mobile.ecs.soton.ac.uk
}

\begin{abstract}
An asynchronous binary DS-CDMA system using random spreading sequences is considered in flat Hoyt fading channels. A new closed-form expression is derived for the conditional characteristic function of the multiple access interference. The exact average BER is expressed as a single numerical integration based on the characteristic function approach. The numerical results obtained from our exact BER analysis are verified by our simulation results and also compared to those obtained by the standard Gaussian approximation.
\end{abstract}

\section{INTRODUCTION}

Code Division Multiple Access (CDMA) has been one of the most successful radio access techniques since the $1990 \mathrm{~s}$ and Direct Sequence (DS) CDMA has been integrated into the third generation mobile systems. The Bit Error Ratio (BER) performance of various DS-CDMA systems has been extensively studied.

For the sake of computational simplicity, the most widely used approach of calculating the average BER of DS-CDMA systems is assuming that the multiple access interference (MAI) is Gaussian distributed or conditional Gaussian distributed based on the Central Limit Theorem (CLT). Various Gaussian approximation techniques have been proposed, such as the Standard Gaussian Approximation (SGA) [1]-[14], the Improved Gaussian Approximation (IGA) [1], [5], [8], [9], [11], [12], [14]-[16], the Simplified IGA (SIGA) [1], [5], [8], [10], [12], [14], as well as the Improved Holtzman Gaussian Approximation (IHGA) [10].

However, the accuracy of various Gaussian approximation techniques has long been criticized [11], especially when the number of users is low and short spreading sequences are used. Hence several exact BER evaluation techniques have also been developed without assuming a Gaussian MAI distribution, such as the series expansion of [11], [12], [17]-[19], or the employment of Moment Generating Functions (MGF) [20] and Characteristic Functions (CF) [1], [2], [10], [14], [17]. These techniques typically achieve more accurate BER evaluation at the cost of a high computation complexity.

The financial support of the EPSRC, UK and the EU in the framework of the NEWCOM, NEXWAY and PHOENIX projects is gratefully acknowledged.
In the existing literature, most results are reported for the BERs of DS-CDMA systems communicating over Additive White Gaussian Noise (AWGN) channels [4]-[9], [11], [12], [14], [15], [17]-[19], [21]-[25], and a few studies also considered Rayleigh channels [1], [11] as well as Nakagami- $m$ channels [2], [3], [10], [16], [20].

As far as the authors are aware, there are no studies on the exact BER analysis of asynchronous DS-CDMA systems using random spreading sequences communicating over Hoyt channels. The Hoyt distribution of Figure 1, also referred to as the Nakagami- $q$ distribution [26], [27], was originally used for modeling radio channels subject to strong ionospheric scintillation, such as satellite links [28], [29]. Recently, it has been used more frequently as one of the important models for the statistical description of fading mobile radio channels [28], [30]. The Rayleigh distribution may be regarded as a special case of the Hoyt distribution [28], [30]. The contribution of this paper is that we provide an exact BER expression, which requires only a single numerical integration with the aid of hypergeometric functions of several variables [31], [32].

This paper is organized as follows. In Section II a general asynchronous DS-CDMA system using BPSK modulation communicating over Hoyt channels is presented. Then in Section III its exact BER performance using random spreading sequences is investigated based on the characteristic function approach. Our numerical results are presented in Section IV and finally our conclusions are provided in Section V.

\section{System Model}

We consider a general asynchronous BPSK modulated DSCDMA system communicating over a Hoyt fading channel and assume that there are $K$ simultaneously transmitting users. Binary random spreading sequences having $L$ chips and a rectangular chip waveform are employed.

\section{A. Hoyt (Nakagami-q) Distribution}

If a complex random variable $\widetilde{h}=h e^{j \varphi}$ is Hoyt distributed, then the Probability Density Function (PDF) of its modulus $h$ 


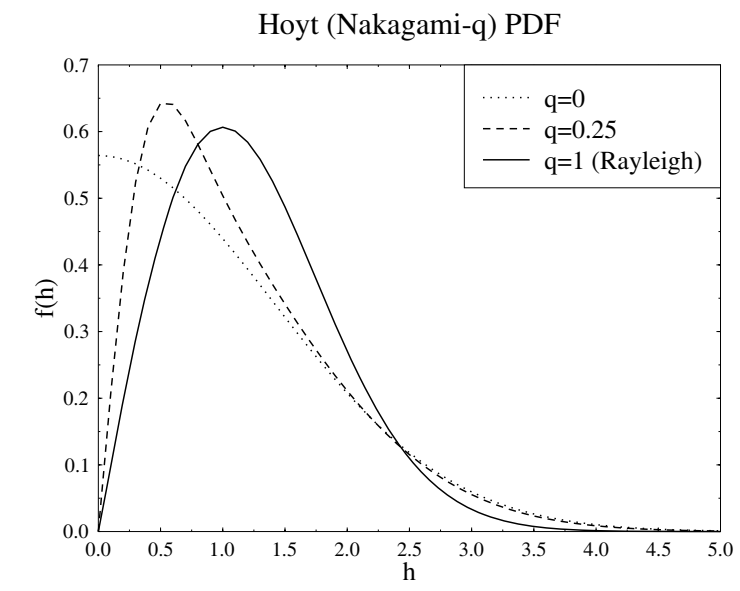

Fig. 1. The PDF of the Hoyt distribution. The Hoyt fading parameter is $q=0,0.25$ and 1 and the average power is $\Omega=2$.

can be expressed as [26]-[28]:

$f(h)=\frac{\left(1+q^{2}\right) h}{q \Omega} \exp \left[-\frac{\left(1+q^{2}\right) h^{2}}{4 q^{2} \Omega}\right] \mathbb{I}_{0}\left[\frac{\left(1-q^{4}\right) h^{2}}{4 q^{2} \Omega}\right], h \geq 0$,

where $\Omega>0$ is the average power, $0 \leq q \leq 1$ is the Hoyt fading parameter and $\mathbb{I}_{0}(x)$ is the zeroth-order modified Bessel function of the first kind [33]. The characteristic function of the modulus $h$ can be shown to be:

$$
\begin{gathered}
\Phi_{h}(\omega)=\frac{2 q}{1+q^{2}} \mathbb{H}_{7}\left[1,1, \frac{1}{2}, \frac{\left(1-q^{2}\right)^{2}}{4\left(1+q^{2}\right)^{2}},-\frac{q^{2} \Omega}{\left(1+q^{2}\right)^{2}} \omega^{2}\right] \\
+j \frac{2 q^{2} \sqrt{\pi \Omega}}{\left(1+q^{2}\right)^{2}} \omega \mathbb{H}_{7}\left[\frac{3}{2}, 1, \frac{3}{2}, \frac{\left(1-q^{2}\right)^{2}}{4\left(1+q^{2}\right)^{2}},-\frac{q^{2} \Omega}{\left(1+q^{2}\right)^{2}} \omega^{2}\right],
\end{gathered}
$$

where $\mathbb{H}_{7}(\alpha, \gamma, \delta, x, y)$ is Horn's confluent hypergeometric function of two variables [31]. Equation 2 provides a closedform expression for the characteristic function of the Hoyt distribution, which is equivalent to the formula given by Table II of [30], but here it is represented in its more compact form, rather than as a sum of infinite series.

The phase of the Hoyt random variable is by no means uniformly distributed [27]. For the sake of facilitating our analysis, we also use an alternative expression of the Hoyt random variable, which is $\widetilde{h}=h_{x}+j h_{y}$ [27], where $h_{x}$ and $h_{y}$ are independent zero-mean Gaussian random variables having a variance of $\sigma_{x}^{2}$ and $\sigma_{y}^{2}$, respectively. The relations of the various parameters are given by:

$$
\begin{aligned}
\Omega & =\sigma_{x}^{2}+\sigma_{y}^{2}, \\
q & =\frac{\sigma_{y}}{\sigma_{x}} .
\end{aligned}
$$

The Hoyt distribution becomes the Rayleigh distribution, when we have $q=1$. Equations 1 and 2 reduce to the PDF and CF of the Rayleigh distribution given by Table II of [30], respectively.

\section{B. Receiver}

The received signal at the input of the coherent correlation receiver is given by:

$r(t)=\Re\left\{\sum_{k=0}^{K} \widetilde{h}_{k} a_{k}\left(t-\tau_{k}\right) b_{k}\left(t-\tau_{k}\right) e^{j\left[\omega_{c}\left(t-\tau_{k}\right)+\theta_{k}\right]}\right\}+\eta(t)$

where $\Re\{\widetilde{x}\}$ denotes the real part of the complex number $\widetilde{x}$. Furthermore, the received complex equivalent signals $\left\{\widetilde{h}_{k}\right\}_{k=0}^{K-1}$ are independent Hoyt distributed random variables having the parameters $\left\{\Omega_{k}, q_{k}\right\}_{k=0}^{K-1}$, or equivalently $\left\{\sigma_{k x}, \sigma_{k y}\right\}_{k=0}^{K-1}$. The carrier's angular frequency $\omega_{c}$ is common to all users, while the carrier phase shift $\left\{\theta_{k}\right\}_{k=0}^{K-1}$ and the time delay $\left\{\tau_{k}\right\}_{k=0}^{K-1}$ are independently and uniformly distributed in $[0,2 \pi)$ and $\left[0, T_{s}\right)$, respectively, where $T_{s}$ is the bit duration. Finally, $\eta(t)$ is the zero-mean stationary Additive White Gaussian Noise (AWGN) having a double-sided power spectral density of $\frac{N_{0}}{2}$. The rectangular pulse having a duration of $T$ is defined as:

$$
p_{T}(t)=\left\{\begin{array}{l}
1, t \in[0, T) \\
0, \text { otherwise. }
\end{array}\right.
$$

Hence the $k$ th user's spreading signal $a_{k}(t)$ and data signal $b_{k}(t)$ can be expressed as:

$$
\begin{aligned}
& a_{k}(t)=\sum_{m=-\infty}^{\infty} a_{k, m} p_{T_{c}}\left(t-m T_{c}\right), \\
& b_{k}(t)=\sum_{m=-\infty}^{\infty} b_{k, m} p_{T_{s}}\left(t-m T_{s}\right),
\end{aligned}
$$

where $T_{c}$ is the chip duration satisfying $T_{s}=L T_{c}$. Both the spreading sequence $\left\{a_{k, m}\right\}_{m=0}^{L-1}$ and the data sequence $\left\{b_{k, m}\right\}_{m=-\infty}^{\infty}$ are mutually independent and symmetrically Bernoulli distributed [34], implying that we have $P\left\{a_{k, m}=\right.$ $\pm 1\}=P\left\{b_{k, m}= \pm 1\right\}=\frac{1}{2}$.

Without loss of generality, we assume that the 0th user's signal is the desired one. If the chip synchronization is perfect, the decision statistic at the output of the coherent correlation receiver is given by:

$$
Z=h_{0} L b_{0,0}+\sum_{k=1}^{K-1} \Re\left\{X_{k} \widetilde{h}_{k} e^{j \Delta_{k}}\right\}+\eta,
$$

where the noise component $\eta$ is a zero-mean Gaussian random variable having a variance of $\sigma_{\eta}^{2}=\frac{N_{0} L}{T_{c}}$, while the phase shift difference $\Delta_{k}=-\omega_{c}\left(\tau_{k}-\tau_{0}\right)+\left(\theta_{k}-\theta_{0}\right)$ between the $k$ th and 0 th user is uniformly distributed in $[0,2 \pi)$. The random variable $X_{k}$ may be further expressed as [1], [6]:

$$
\begin{aligned}
X_{k}= & \sum_{m=0}^{L-2} Y_{k, m}\left[\left(1-\nu_{k}\right)+a_{0, m} a_{0, m+1} \nu_{k}\right] \\
& +Y_{k, L-1} \nu_{k}+Y_{k, L}\left(1-\nu_{k}\right),
\end{aligned}
$$

where the $(L+1)$ random variables $\left\{Y_{k, m}\right\}_{m=0}^{L}$ are mutually independent and symmetric Bernoulli distributed, conditioned on the 0th user's spreading sequence $\left\{a_{0, m}\right\}_{m=0}^{L-1}$. Furthermore, the relative chip shifts $\left\{\nu_{k}\right\}_{k=1}^{K-1}$ between the $k$ th and 0 th user 
normalized by the chip duration are mutually independent and uniformly distributed in $[0,1)[1],[6]$.

\section{BER ANALYSIS}

Let $B$ and $A$ denote the number of chip boundaries both with and without chip-value transitions within the 0th user's spreading sequence, respectively, and define two sets $\mathcal{A}$ and $\mathcal{B}$ as follows [1], [6]:

$$
\begin{aligned}
& \mathcal{A}=\{-A,-(A-2), \ldots, A-2, A\}, \\
& \mathcal{B}=\{-B,-(B-2), \ldots, B-2, B\} .
\end{aligned}
$$

Then we have $A+B=L-1$ and the Co-Channel Interference (CCI) $I_{k}=\Re\left\{X_{k} \widetilde{h}_{k} e^{j \Delta_{k}}\right\}$ imposed by the different interferers is mutually independent, conditioned on $B$ [1], [6]. For the sake of simplicity, we will only consider the characteristic function range spanning over $\omega \geq 0$ in our later discussions in the context of Equation 18. Nevertheless, the characteristic function range spanning over $\omega \leq 0$ can be readily derived from the range spanning over $\omega \geq 0$ by exploiting the following property of the characteristic function [34] $\Phi(-\omega)=\Phi^{*}(\omega)$, where $\Phi^{*}(\omega)$ denotes the complex conjugate of $\Phi(\omega)$.

Upon exploiting the alternative expression of the Hoyt random variable $\widetilde{h}_{k}$ in Section II-A, it can be readily shown that $I_{k}$ conditioned on $X_{k}$ and $\Delta_{k}$ is a zero-mean Gaussian random variable having a variance of $\sigma_{k x}^{2} \cos ^{2} \Delta_{k}+\sigma_{k y}^{2} \sin ^{2} \Delta_{k}$. Hence we have the characteristic function of $I_{k}$ conditioned on $X_{k}$ and $\Delta_{k}$ in the following form:

$\Phi_{I_{k} \mid X_{k}, \Delta_{k}}(\omega)=\exp \left[-\frac{1}{2}\left(\sigma_{k x}^{2} \cos ^{2} \Delta_{k}+\sigma_{k y}^{2} \sin ^{2} \Delta_{k}\right) X_{k}^{2} \omega^{2}\right]$

Applying the integral identity of Equation 3.339 in [33], we arrive at the characteristic function of $I_{k}$ conditioned on $X_{k}$ by averaging $\Phi_{I_{k} \mid X_{k}, \Delta_{k}}(\omega)$ over $\Delta_{k} \in[0,2 \pi)$ in the form of:

$$
\begin{aligned}
\Phi_{I_{k} \mid X_{k}}(\omega)= & \exp \left[-\frac{1}{4}\left(\sigma_{k x}^{2}+\sigma_{k y}^{2}\right) X_{k}^{2} \omega^{2}\right] \\
& \times \mathbb{I}_{0}\left[\frac{1}{4}\left(\sigma_{k x}^{2}-\sigma_{k y}^{2}\right) X_{k}^{2} \omega^{2}\right] .
\end{aligned}
$$

Upon averaging $\Phi_{I_{k} \mid X_{k}}(\omega)$ over $\left\{Y_{k, m}\right\}_{m=0}^{L}$ and $\nu_{k}$, we get the characteristic function of $I_{k}$ conditioned on $\mathrm{B}$ [1] in the following form:

$$
\begin{gathered}
\Phi_{I_{k} \mid B}(\omega)=2^{-(L+1)} \sum_{d_{1} \in \mathcal{A}} \sum_{d_{2} \in \mathcal{B}}\left(\begin{array}{c}
A \\
\frac{d_{1}+A}{2}
\end{array}\right)\left(\begin{array}{c}
B \\
\frac{d_{2}+B}{2}
\end{array}\right) \\
\times \sum_{Y_{k, L-1}, Y_{k, L} \in\{ \pm 1\}} \Phi_{I_{k} \mid \lambda_{0}, \lambda_{1}}(\omega),
\end{gathered}
$$

where the coefficients $\lambda_{0}$ and $\lambda_{1}$ are defined as:

$$
\begin{aligned}
& \lambda_{0}=d_{1}+d_{2}+Y_{k, L} \\
& \lambda_{1}=-2 d_{2}+Y_{k, L-1}-Y_{k, L} .
\end{aligned}
$$

When invoking the series expansions of Equations 1.211-1 and $8.477-1$ of [33], the conditional characteristic function $\Phi_{I_{k} \mid \lambda_{0}, \lambda_{1}}(\omega)$ may be expressed in the form of Equation 17

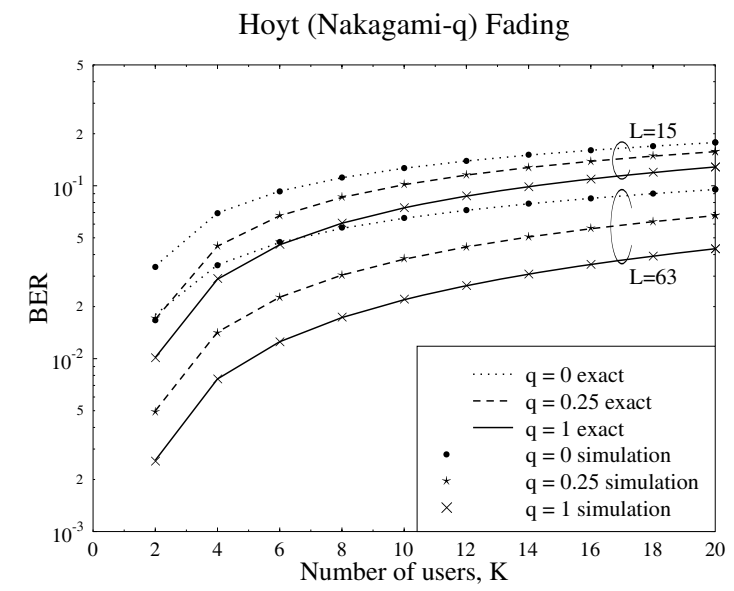

Fig. 2. BER versus the number of users $K$ in an asynchronous DSCDMA system using random spreading sequences and BPSK modulation communicating over Hoyt channels. The length of the random spreading sequences is $L=15$ and 63 . The Hoyt fading parameter is $q=0,0.25$ and 1 , which is common to all users. The average power of all users at the receiver is equal and the background noise is ignored, i.e. we have $\gamma_{\mathrm{SNR}}=\infty$.

seen at the top of the next page. When $q=1$, i.e. when we experience Rayleigh fading, Equation 17 reduces to the results of [1].

Applying Parseval's theorem [30] and exploiting the fact that the CCI contribution $\left\{I_{k}\right\}$ conditioned on $B$ is mutually independent [1], [6], we arrive at the 0th user's BER conditioned on $B$ as follows:

$$
P_{e \mid B}=\frac{1}{2}-\frac{1}{\pi} \int_{0}^{\infty} \frac{1}{\omega} \Phi_{\eta}(\omega) \Im\left\{\Phi_{h_{0}}(\omega L)\right\} \prod_{k=1}^{K-1} \Phi_{I_{k}}(\omega) \mathrm{d} \omega,
$$

where $\Phi_{\eta}(\omega)$ is the characteristic function of the noise component $\eta$ and $\Im\left\{\Phi_{h_{0}}(\omega)\right\}$ is the imaginary part of the characteristic function of $h_{0}$, which was given by Equation 2 .

Finally, we arrive at the overall average BER by averaging $P_{e \mid B}$ over all spreading sequences in the form of:

$$
P_{e}=2^{-(L-1)} \sum_{B=0}^{L-1}\left(\begin{array}{c}
L-1 \\
B
\end{array}\right) P_{e \mid B}
$$

\section{NUMERICAL RESUlts}

In this section we will verify the accuracy of our exact BER analysis provided in Section III and demonstrate the limited accuracy of the SGA method by simulations.

Figures 2 and 3 illustrate the average BER performance versus the number of users, when the effects of background noise are ignored. Figure 2 compares the results obtained from our exact BER analysis to our simulation results and shows that they match very well both for different spreading sequence lengths and for various Hoyt fading parameters. On the other hand, Figure 3 compares the results obtained using the SGA to our simulation results and demonstrates that the SGA overestimates the average BER, especially in the scenarios where 


$$
\Phi_{I_{k} \mid \lambda_{0}, \lambda_{1}}(\omega)= \begin{cases}\left.\left.\frac{x}{\lambda_{1}} \mathbb{F}_{1: 0 ; 1}^{1: 0 ; 0}\left(\left[\left(\frac{1}{2}\right): 1,2\right]:-;-; \frac{3}{2}\right): 1,2\right]:-;[(1): 1] ;-\frac{1}{4}\left(\sigma_{k x}^{2}+\sigma_{k y}^{2}\right) \omega^{2} x^{2}, \frac{1}{64}\left(\sigma_{k x}^{2}-\sigma_{k y}^{2}\right)^{2} \omega^{4} x^{4}\right)\left.\right|_{\lambda_{0}} ^{\lambda_{0}+\lambda_{1}}, \lambda_{1} \neq 0 \\ \exp \left[-\frac{1}{4}\left(\sigma_{k x}^{2}+\sigma_{k y}^{2}\right) \lambda_{0}^{2} \omega^{2}\right] \mathbb{I}_{0}\left[\frac{1}{4}\left(\sigma_{k x}^{2}-\sigma_{k y}^{2}\right) \lambda_{0}^{2} \omega^{2}\right], & \lambda_{1}=0\end{cases}
$$

where $\mathbb{F}_{C: D^{(1)} ; \ldots ; D^{(n)}}^{A: B^{(1)} ; \ldots ; B^{(n)}}\left(\left[\left((a): \theta^{(1)}, \ldots, \theta^{(n)}\right]:\left[\left(b^{(1)}\right): \phi^{(1)}\right] ; \ldots ;\left[\left(b^{(n)}\right): \phi^{(n)}\right] ; \psi^{(1)}, \ldots, \psi^{(n)}\right]:\left[\left(d^{(1)}\right): \delta^{(1)}\right] ; \ldots ;\left[\left(d^{(n)}\right): \delta^{(n)}\right] ; x_{1}, \ldots, x_{n}\right)$ is the generalized Lauricella function of $n$ variables defined as Equations $21-23$ of [32] and $\left.f(x)\right|_{x_{1}} ^{x_{2}}=f\left(x_{2}\right)-f\left(x_{1}\right)$.

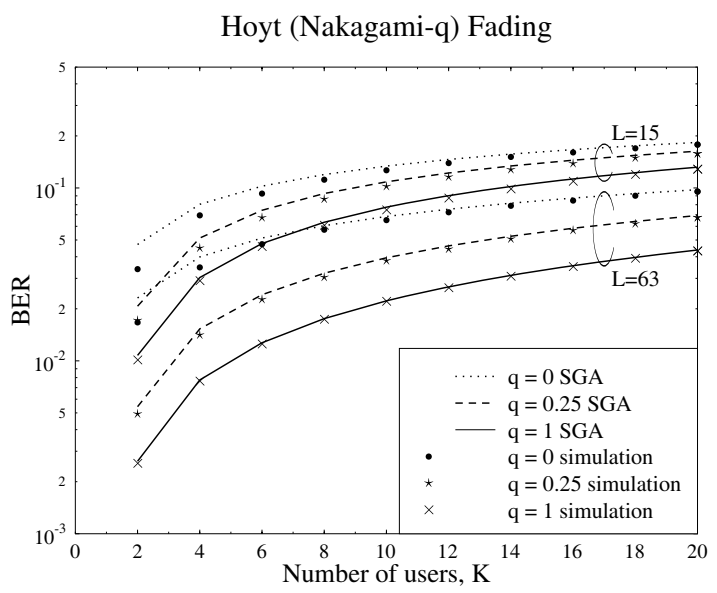

Fig. 3. BER versus the number of users $K$ in an asynchronous DSCDMA system using random spreading sequences and BPSK modulation communicating over Hoyt channels. The length of the random spreading sequences is $L=15$ and 63 . The Hoyt fading parameter is $q=0,0.25$ and 1. The average power of all users at the receiver is equal and the background noise is ignored, i.e. we have $\gamma_{\mathrm{SNR}}=\infty$.

either there is a limited number of interferers, or when the Hoyt fading parameter $q$ is small or when short spreading sequences are used.

Figures 4 and 5 illustrate the average BER performance versus the per-bit SNR, when the number of users is $K=4$. Figure 4 compares the results obtained from our exact BER analysis to our simulation results and shows that they match well both for different spreading sequence lengths and for various Hoyt fading parameters. On the other hand, Figure 5 compares the results obtained by the SGA to our simulation results and demonstrates that the SGA still fails to accurately evaluate the average BER performance, particularly when the SNR is high, the Hoyt fading parameter $q$ is low and when short spreading sequences are used.

\section{CONCLUSion}

An exact expression has been derived for calculating the average BER of an asynchronous DS-CDMA system using random spreading sequences and BPSK modulation for communicating over Hoyt fading channels. It is based on the characteristic function and requires only a single numerical

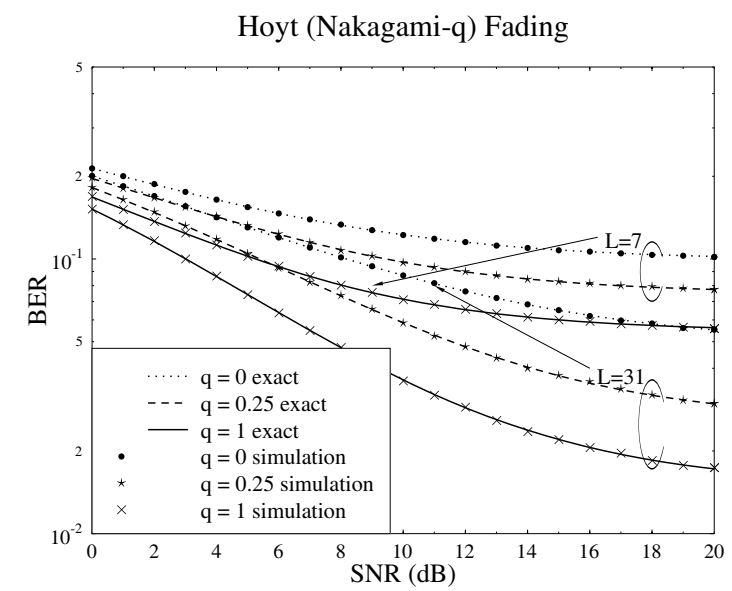

Fig. 4. BER versus per-bit SNR in an asynchronous DS-CDMA system using random spreading sequences and BPSK modulation communicating over Hoyt channels. The length of the random spreading sequences is $L=7$ and 31 . The Hoyt fading parameter is $q=0,0.25$ and 1 . The average power of all users at the receiver is equal. The number of users is $K=4$.

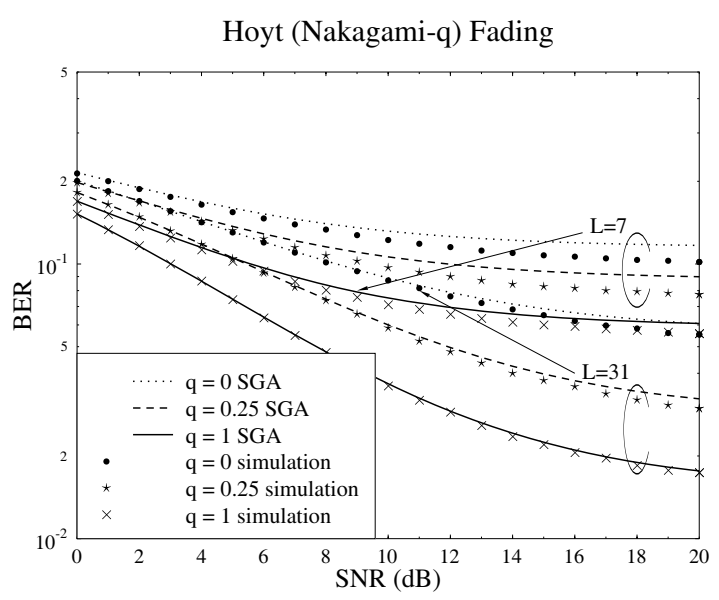

Fig. 5. BER versus per-bit SNR in an asynchronous DS-CDMA system using random spreading sequences and BPSK modulation communicating over Hoyt channels. The length of the random spreading sequences is $L=7$ and 31 . The Hoyt fading parameter is $q=0,0.25$ and 1 . The average power of all users at the receiver is equal. The number of users is $K=4$. 
integration. Furthermore, a new closed-form expression was provided for the conditional characteristic function of the interfering signal with the aid of the generalized Lauricella function in $n$ variables [32]. Since the Rayleigh distribution is a special case of the Hoyt distribution, the results obtained by [1] may also be regarded as a special case of our results. Our simulation results verified the accuracy of our exact BER analysis for various combinations of the spreading sequence length and the Hoyt fading parameter. By contrast, the SGA over-estimates the average BER, especially when either there is a low number of interferers, or the SNR is high, the Hoyt fading parameter happens to be low or and short spreading sequences are used.

\section{REFERENCES}

[1] J. Cheng and N. Beaulieu, "Accurate DS-CDMA Bit-Error Probability Calculation in Rayleigh Fading," IEEE Transactions on Wireless Communications, vol. 1, no. 1, pp. 3-15, January 2002.

[2] — - "Precise Bit Error Rate Calculation for Asynchronous DS-CDMA in Nakagami Fading," in IEEE Global Telecommunications Conference, vol. 2, San Francisco, CA, 27 September-1 December 2000, pp. 980984.

[3] T. Eng and L. B. Milstein, "Coherent DS-CDMA Performance in Nakagami Multipath Fading," IEEE Transactions on Communications, vol. 43, no. 2/3/4, pp. 1134-1143, February/March/April 1995.

[4] E. Geraniotis and B. Ghaffari, "Performance of Binary and Quaternary Direct-Sequence Spread-Spectrum Multiple-Access Systems with Random Signature Sequences," IEEE Transactions on Communications, vol. 39, no. 5, pp. 713-724, May 1991.

[5] J. M. Holtzman, "A Simple, Accurate Method to Calculate SpreadSpectrum Multiple-Access Error Probabilities," IEEE Transactions on Communications, vol. 40, no. 3, pp. 461-464, March 1992.

[6] J. S. Lehnert and M. B. Pursley, "Error Probabilities for Binary DirectSequence Spread-Spectrum Communications with Random Signature Sequences," IEEE Transactions on Communications, vol. 35, no. 1, pp. 87-98, January 1987.

[7] — "Multipath Diversity Reception of Spread-Spectrum MultipleAccess Communications," IEEE Transactions on Communications, vol. 35, no. 11, pp. 1189-1198, November 1987

[8] T. M. Lok and J. S. Lehnert, "Error Probabilities for Generalized Quadriphase DS/SSMA Communication Systems with Random Signature Sequences," IEEE Transactions on Communications, vol. 44, no. 7, pp. 876-885, July 1996

[9] J. R. K. Morrow and J. S. Lehnert, "Bit-to-Bit Error Dependence in Slotted DS/SSMA Packet Systems with Random Signature Sequences," IEEE Transactions on Communications, vol. 37, no. 10, pp. 1052-1061, October 1989.

[10] K. Sivanesan and N. C. Beaulieu, "Performance Analysis of Bandlimited DS-CDMA Systems in Nakagami Fading," in IEEE International Conference on Communications, vol. 1, Paris, France, 20-24 June 2004, pp. $400-404$.

[11] M. O. Sunay and P. J. McLane, "Calculating Error Probabilities for DS-CDMA Systems: When Not to Use the Gaussian Approximation," in IEEE Global Telecommunications Conference, vol. 3, London, UK, 18-22 November 1996, pp. 1744-1749.

[12] _ - "Sensitivity of a DS CDMA System with Long PN Sequences to Synchronization Errors," in IEEE International Conference on Cоттиnications, vol. 2, Seattle, WA, USA, 18-22 June 1995, pp. 1029-1035.

[13] C. Unger and G. P. Fettweis, "Analysis of the RAKE Receiver Performance in Low Spreading Gain DS/SS Systems," in IEEE Global Telecommunications Conference 2002, vol. 1, 17-21 November 2002, pp. 830-834.

[14] Y. C. Yoon, "Quadriphase DS-CDMA with Pulse Shaping and the Accuracy of the Gaussian Approximation for Matched Filter Receiver Performance Analysis," IEEE Transactions on Wireless Communications, vol. 1, no. 4, pp. 761-768, October 2002.

[15] A. Mirbagheri and Y. C. Yoon, "Performance Analysis of a Linear MMSE Receiver for Bandlimited Random-CDMA Using Quadriphase Spreading over Multipath Channels," IEEE Transactions on Wireless Communications, vol. 3, no. 4, pp. 1053-1066, July 2004.
[16] K. Sivanesan and N. C. Beaulieu "Accurate BER Analysis of Bandlimited DS-CDMA System with EGC and SC Diversity over Nakagami Fading Channels," in IEEE Wireless Communications and Networking Conference, vol. 2, New Orleans, Louisiana, USA, 13-17 March 2005, pp. $956-960$.

[17] E. A. Geraniotis and M. B. Pursley, "Error Probability for DirectSequence Spread-Spectrum Multiple-Access Communications-Part II: Approximations," IEEE Transactions on Communications, vol. 30, no. 5 , pp. 985-995, May 1982 .

[18] M. O. Sunay and P. J. McLane, "Comparison of Biphase Spreading to Quadriphase Spreading in DS CDMA Systems that Employ Long PN Sequences," in Sixth IEEE International Symposium on Personal, Indoor and Mobile Radio Communications, vol. 1, Toronto, Canada, 27-29 September 1995, pp. 237-242.

[19] _ - "Effects of Carrier Phase and Chip Timing Errors on the Capacity of a Quadriphase Spread BPSK Modulated DS-CDMA System," in IEEE Global Telecommunications Conference, vol. 2, Singapore, 13-17 November 1995, pp. 1114-1120.

[20] Q. Shi and M. Latva-Aho, "Accurate Bit-Error Rate Evaluation for Synchronous MC-CDMA over Nakagami- $m$-Fading Channels Using Moment Generating Functions," IEEE Transactions on Wireless Communications, vol. 4, no. 2, pp. 422-433, March 2005.

[21] F. D. Garber and M. B. Pursley, "Performance of Offset Quadriphase Spread-Spectrum Multiple-Access Communications," IEEE Transactions on Communications, vol. 29, no. 3, pp. 305-314, March 1981.

[22] R. T. Hsu and J. S. Lehnert, "A Characterization of Multiple-Access Interference in Generalized Quadriphase Spread-Spectrum Communications," IEEE Transactions on Communications, vol. 42, no. 2/3/4, pp. 2001-2010, FEBRUARY/MARCH/APRIL 1994.

[23] D. Laforgia, A. Luvison, and V. Zingarelli, "Bit Error Rate Evaluation for Spread-Spectrum Multiple-Access Systems," IEEE Transactions on Communications, vol. 32, no. 6, pp. 660-669, June 1984

[24] M. B. Pursley, D. V. Sarwate, and W. E. Stark, "Error Probability for Direct-Sequence Spread-Spectrum Multiple-Access CommunicationsPart I: Upper and Lower Bounds," IEEE Transactions on Communications, vol. 30, no. 5, pp. 975-984, May 1982.

[25] M. B. Pursley, "Performance Evaluation for Phase-Coded SpreadSpectrum Multiple-Access Communication-Part I: System Analysis," IEEE Transactions on Communications, vol. 25, no. 8, pp. 795-799, August 1977.

[26] M. Nakagami, "The $m$-Distribution - A General Formula of Intensity Distribution of Rapid Fading," in Statistical Methods in Radio Wave Propagation, W. C. Hoffman, Ed. London: Pergamon Press, 1960, pp. 3-36.

[27] R. S. Hoyt, "Probability Functions for the Modulus and Angle of the Normal Complex Variate," Bell System Technical Journal, vol. 26, pp. 318-359, April 1947.

[28] M. K. Simon and M.-S. Alouini, "A Unified Approach to the Performance Analysis of Digital Communication over Generalized Fading Channels," IEEE Proceedings, vol. 86, no. 9, pp. 1860-1877, September 1998.

[29] B. Chytil, "The Distribution of Amplitude Scintillation and the Conversion of Scintillation Indices," Journal of Atmospheric and Terrestrial Physics, vol. 29, pp. 1175-1777, September 1967.

[30] A. Annamalai, C. Tellambura, and V. K. Bhargava, "Equal-Gain Diversity Receiver Performance in Wireless Channels," IEEE Transactions on Communications, vol. 48, no. 10, pp. 1732-1745, October 2000.

[31] A. Erdelyi, W. Magnus, F. Oberhettinger, and F. G. Tricomi, Eds., Higher Transcendental Functions. New York, Toronto and London: McGrawHill Book Company, Inc., 1953, vol. 1 .

[32] H. M. Srivastava and P. W. Karlsson, Multiple Gaussian Hypergeometric Series. Ellis Horwood, Ltd., 1985.

[33] I. S. Gradshteyn and I. M. Ryzhik, Table of Integrals, Series, and Products, 6th ed., A. Jeffrey and D. Zwillinger, Eds. Academic Press, 2000.

[34] A. Papoulis, Probability, Random Variables, and Stochastic Processes, 3rd ed. McGraw-Hill, Inc., 1991. 